\title{
Vibrational coherence in ultrafast electron transfer reaction observed by broadband transient absorption spectroscopy
}

\author{
Yusuke Yoneda ${ }^{1, *}$, Bryan Kudisch ${ }^{2}$, Shahnawaz Rafiq $^{2}$, Margherita Maiuri ${ }^{2}$, Gregory \\ Scholes $^{2}$, and Hiroshi Miyasaka ${ }^{1}$ \\ ${ }^{1}$ Graduate School of Engineering Science, Osaka University, 1 -3 Machikaneyama, Toyonaka, Japan \\ ${ }^{2}$ Department of Chemistry, Princeton University, Princeton, USA
}

\begin{abstract}
Photoinduced electron transfer (ET) is one of the most important processes in light energy conversion systems. Marcus theory is one of the most famous frameworks on ET reaction in condensed phase. In this theory, reorganization of surrounding media is regarded as a main reaction coordinate. However, ultrafast ET beyond Marcus' framework has been reported in various systems. To elucidate the role of molecular vibration in ultrafast ET, we have investigated the ET dynamics between a naphthacene dye and aniline derivatives by means of broadband transient absorption spectroscopy. Coherent wavepacket motions of naphthacene dye with frequencies of $300-1600 \mathrm{~cm}^{-1}$ were observed in time domain. The vibrational coherence of $310 \mathrm{~cm}^{-1}$ mode was reduced with increasing electron transfer rate, suggesting this vibration is coupled to electron transfer reaction.
\end{abstract}

\section{Introduction}

Photoinduced electron transfer (ET) reaction is one of the most important fundamental processes in light energy conversion systems such as solar cell, photosynthesis and artificial photosynthesis. To increase the efficiency of ET reaction, a large reaction rate is required because the reaction takes place in the electronically excited state with a finite lifetime.

Marcus theory is one of the most famous frameworks on the ET reaction in condensed phase. In this theory, reorganization of surrounding media is regarded as a main reaction coordinate leading to the bell-shaped relation between the rate constant and the energy gap. Although this postulation is valid for the equilibrium system, ultrafast ET reactions beyond the Marcus' framework have been reported in various systems. Recently, ultrafast ET in photosynthetic reaction center is suggested to involve vibrionic coherence [1,2].

To elucidate the role of intra/inter molecular vibrations in ultrafast ET reaction, we have investigated ultrafast ET system, a dye dissolved in electron donating solvents, by means of broadband transient absorption (TA) spectroscopy. We have used five aniline derivatives (Scheme 1) for electron donating solvents to systematically investigate the effect of the coherent wavepacket motion in the ET process. For a solute molecule, 5,12-

\footnotetext{
* Corresponding author: yoneda@laser.chem.es.osaka-u.ac.jp
} 
bis(phenylethynyl)-naphthacene (BPEN) was chosen because it has strong vibrational structure in absorption spectrum and thus is expected to exhibit strong coherent wavepacket motions.

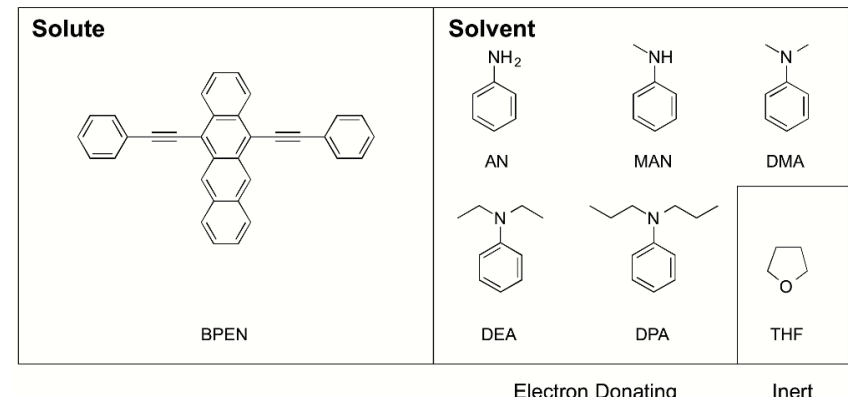

Scheme 1. Molecular structure of 5,12-bis(phenylethynyl)-naphthacene (BPEN) and Aniline (AN), $N$-methylaniline (MAN), $N, N$-dimethylaniline (DMA), $N, N$-diethylaniline (DEA), $N, N$ dipropylaniline (DPA) and tetrahydrofurane (THF).

\section{Experimental methods}

For broadband transient absorption spectroscopy measurement, the light source was regeneratively amplified Ti:sapphire laser (Solstice, Spectra Physics) and used to pump homebuild nonlinear optical parametric amplifier (NOPA). The desired NOPA pulse spectrum was compressed by chirped mirrors. Pulse duration was estimated to be $10 \mathrm{fs}$ by polarization-gated frequency resolved optical gating (PG-FROG). The polarization between pump and probe pulse was set as parallel.

\section{Results and discussion}

TA spectra of electron-accepting dye, BPEN, in an inert solvent, THF (Fig. 1a), show ground state bleach (GSB) at 550-600 nm, stimulated emission (SE) at 620-630 nm and excited state absorption (ESA) which overlaps entire spectral range of 540-660 nm, as observed in previous study [3]. In the electron-donating solvents, SE of BPEN was quenched by ultrafast ET from the solvent to BPEN, as indicated by the disappearance of $\mathrm{SE}$ signal around $625 \mathrm{~nm}$ in Fig. 1c. A new positive signal at 600-660 nm appears, which is ascribed to the formation of ET product (BPEN anion).

Coherent broadband pump pulse generates coherent superposition of vibrational levelscalled as wavepacket, which create modulations in time-domain. The oscillatory responses were extracted from TA signals at each probe wavelength after subtracting slow dynamics and the residuals were Fourier transformed to frequency domain spectra (Fig. 1b, d). In THF, all the frequencies, except for $913 \mathrm{~cm}^{-1}$, can be assigned to BPEN from the comparison with frequencies obtained for the THF solvent. Frequencies monitored at probe wavelengths $>600 \mathrm{~nm}$ can be safely assigned to the wavepacket motion in the excited state because there is no contribution from GSB in this wavelength range. Eight clear vibrational bands were observed in this spectral region with frequencies of $300-1600 \mathrm{~cm}^{-1}$.

Dephasing time constants of each vibrational mode at $620 \mathrm{~nm}$ were estimated by fitting time domain signals obtained with inverse Fourier filtering procedure using super-Gaussian function [4]. The dephasing time of the vibrational coherence oscillating at $310 \mathrm{~cm}^{-1}$ becomes faster with an increase in the ET rate (Fig. 2). This result strongly suggests that coherence of $310 \mathrm{~cm}^{-1}$ mode is coupled to the ultrafast ET process. The vibrational mode 
can be assigned to in-plane stretching mode from the comparison with the result of TDDFT calculation, also suggesting this mode can effectively modulate the electronic coupling between BPEN excited state and ET state.
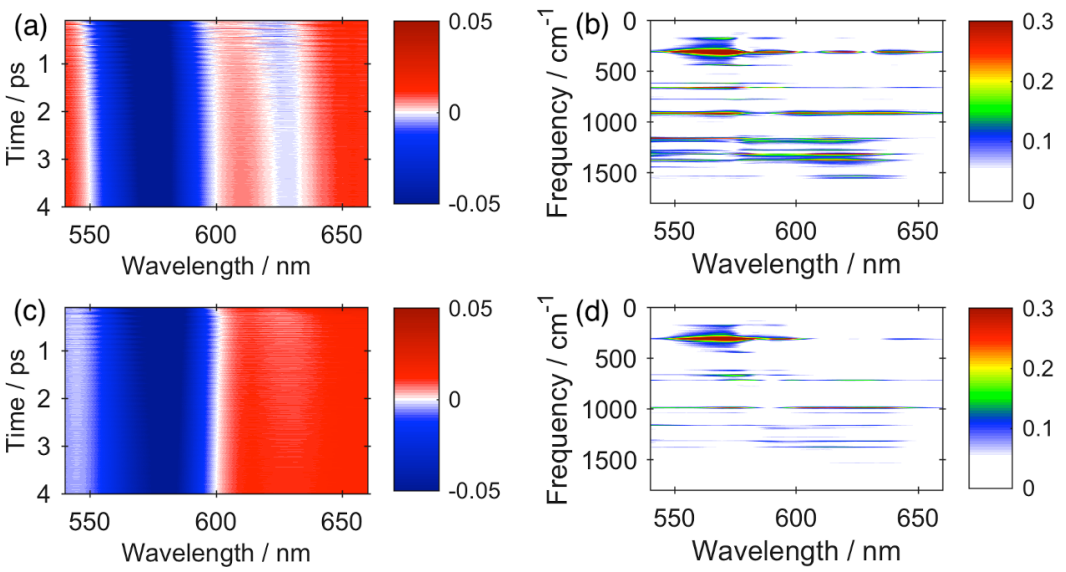

Fig. 1. Transient absorption map of BPEN in (a) THF and (b) DEA. Amplitude of Fourier transformed spectra of the residuals obtained by subtracting slow varying dynamics of TA signals of BPEN in (c) THF and (d) DEA.

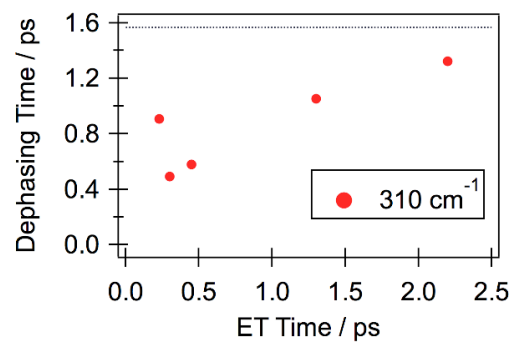

Fig. 2. Dephasing time constant of $310 \mathrm{~cm}^{-1}$ mode at $620 \mathrm{~nm}$ in each electron donating solvent plotted against electron transfer time constant. Dashed line represents dephasing time constant in THF.

This work was supported by JSPS KAKENHI Grant Numbers JP16J00627 and JP26107002.

\section{References}

1. F. D. Fuller, J. Pan, A. Gelzinis, V. Butkus, S. S. Senlik, D. E. Wilcox, C. F. Yocum, L. Valkunas, D. Abramavicius, J. P. Ogilvie, Nature Chemistry 6, 706 (2014)

2. E. Romero, R. Augulis, V. I. Novoderezhkin, M. Ferretti, J. Thieme, D. Zigmantas, R. van Grondelle, Nature Physics 10, 676 (2014)

3. Y. Yoneda, S. Nambu, E. Takeuchi, Y. Nagasawa, H. Miyasaka, J. Photochem. Photobiol. A Chem. 313, 79 (2015)

4. S. Rafiq, G. D. Scholes, Chem. Phys. Lett. 683, 500 (2017) 